\title{
Prevalência de isoimunização Rh materna em maternidade pública do Amazonas entre 2018 e 2020
}

\author{
Prevalence of maternal $\mathrm{Rh}$ isoimmunization in public maternity hospitals in Amazonas \\ between 2018 and 2020
}
Prevalencia de la isoinmunización Rh materna en las maternidades públicas de Amazonas entre 2018 y 2020

Aida Pinto Fernandes ${ }^{1 *}$, Claudia Marques de Oliveira Soeiro ${ }^{1}$, Fernanda Araújo Ribeiro ${ }^{1}$, Kassya da Silva Rebelo ${ }^{1}$, Glauber Palma de Oliveira ${ }^{2}$.

\section{RESUMO}

Objetivo: Analisar a prevalência de isoimunização Rh materna no período de 2018 a 2020 em maternidade do Amazonas. Métodos: Foi realizado um estudo observacional e descritivo retrospectivo de casos de sensibilização materna a $R h(D)$ atendidos em uma maternidade pública na cidade de Manaus, Amazonas. Foram utilizados dados secundários a partir do prontuário para análise de variáveis como tipagem sanguínea e fator RhD, idade, raça, idade gestacional, paridade, testes de controle Coombs. Esses prontuários estavam catalogados no Serviço de Atendimento Médico e de Emergência (SAME) no período de estudo. Resultados: A prevalência foi de $1,49 \%$ dos casos notificados. A média de idade da gestante foi de 25,17 anos, sendo $63 \%$ das pacientes de cor parda. A tipagem sanguínea, foi de $61,2 \%$ das mulheres do tipo $O$ negativo e os recém-nascidos, $41,5 \% O$ positivo. A média de consultas de pré-natal foi de apenas 5,3 consultas. Conclusão: A taxa de prevalência encontrada, apesar de baixa, poderia ser ainda menor, uma vez que na maioria das mulheres da Região Norte outros tipos sanguíneos são mais prevalentes. O pré-natal com número reduzido de consultas pode ter sido fator responsável pela falha da prevenção da Doença Hemolítica perinatal no Estado.

Palavras-chave: Isoimunização Rh, Imunoglobulina rho(D), Assistência pré-natal.

\begin{abstract}
Objective: To analyze the prevalence of maternal $\mathrm{Rh}$ isoimmunization from 2018 to 2020 in a maternity hospital in Amazonas. Methods: An observational and descriptive retrospective study of cases of maternal $\mathrm{Rh}(\mathrm{D})$ sensitization attended in a public maternity hospital in the city of Manaus, Amazonas, Brazil, was conducted. Secondary data from medical records were used to analyze variables such as blood typing and $\mathrm{RhD}$ factor, age, race, gestational age, parity, Coombs control tests. These medical records were catalogued in the Medical and Emergency Care Service (SAME) during the study period. Results: The prevalence was $1.49 \%$ of reported cases. The mean age of pregnant women was 25.17 years, and $63 \%$ of the patients were brown. The blood typing was $61.2 \%$ of the women were $O$ negative and the newborns $41.5 \%$ were $O$ positive. The mean number of prenatal visits was only 5.3. Conclusion: The prevalence rate found, although low, could be even lower, since in most women in the North Region other blood types are more prevalent. The low number of prenatal consultations may have been a factor responsible for the failure to prevent perinatal hemolytic disease in the state.
\end{abstract}

Keywords: Rh isoimmunization, Rho(D) immunoglobulin, Prenatal care.

1 Universidade do Estado do Amazonas (UEA), Manaus - AM. *E-mail: aidapfernandes@gmail.com

2 Universidade Nilton Lins (UNL), Manaus - AM.

SUBMETIDO EM: 9/2021

ACEITO EM: 9/2021

PUBLICADO EM: 9/2021 


\section{RESUMEN}

Objetivo: Analizar la prevalencia de isoimunización Rh materna en el período de 2018 a 2020 en la maternidad del Amazonas. Métodos: Realizamos un estudio retrospectivo observacional y descriptivo de los casos de sensibilización al $\mathrm{Rh}(\mathrm{D})$ materno atendidos. Se utilizaron datos secundarios a partir del prontuario para el análisis de variables como el tipo de sangre y el factor $\mathrm{RhD}$, la edad, la raza, la edad gestacional, la paridad y las pruebas de control de Coombs. Estas historias clínicas fueron catalogadas en el Servicio de Atención Médica y de Urgencias (SAME) en el periodo de estudio. Resultados: La prevalencia fue del 1,49\% de los casos declarados. La edad media de las embarazadas era de 25,17 años, y el 63\% de las pacientes eran morenas. La tipificación sanguínea, fue del $61,2 \%$ de las mujeres tipo $O$ negativo y los recién nacidos, del $41,5 \%$ O positivo. La media de consultas prenatales fue de sólo 5,3 consultas. Conclusión: La tasa de prevalencia encontrada, podría ser incluso menor, ya que en la mayoría de las mujeres de la Región Norte son más frecuentes otros tipos de sangre. El bajo número de consultas prenatales puede haber sido un factor responsable del fracaso en la prevención de la enfermedad hemolítica perinatal.

Palabras clave: Isoinmunización Rh, Inmunoglobulina rho(D), Atención prenatal.

\section{INTRODUÇÃO}

Na prática obstétrica, dentre os vários grupos sanguíneos, o ABO e RhD são de suma importância clínica, pois pode ocorrer a sensibilização em mulheres $\mathrm{RhD}$ negativas a partir de conceptos $\mathrm{RhD}$ positivos (herança paterna) que culmina na produção de anticorpos anti-D. Tais anticorpos, ao atravessarem a barreira placentária podem levar à anemia hemolítica perinatal (DHPN) a partir do processo de hemólise de eritrócitos fetais. A formação dos anticorpos maternos em um antígeno fetal é um evento chamado de isoimunização. Eles são formados quando eritrócitos fetais ao expressarem determinados antígenos em suas hemácias, que são ausentes na gestante, têm contato com o sangue materno (NASSAR GN e WEHBE C, 2020). Esses antígenos na superfície dos eritrócitos possuem uma alta variedade e pertencem ao fator Rhesus (fator Rh), sendo o antígeno $D$ o principal responsável pela doença $R$ h devido à sua alta imunogenicidade (SARWAR A e SRIDHAR DC, 2020).

Sendo assim, o feto passa a ser um "invasor" no corpo da grávida e seus anticorpos passam a atacar os eritrócitos fetais, levando a diversas consequências ao bebê através do processo de hemólise e pode gerar quadros profundos de anemia. Tais complicações dependem muito do grau de sensibilização materna (NASSAR GN e WEHBE C, 2020). Quanto aos mecanismos de ação dos anticorpos anti-D foram elucidados a liberação acelerada de células Rh positivas, o mascaramento do determinante antigênico, a inibição de células dendríticas imaturas, dentre outras. Porém, o mecanismo mais aceito atualmente é a destruição acelerada de glóbulos vermelhos Rh-positivo (MC BRAIN RD, et al., 2015).

Por isso, no início da década de 1960 foi estabelecido o protocolo clínico de imunização passiva de gestantes $R h(D)$ negativos com imunoglobulina anti-Rh como medida de prevenção, não obstante seu mecanismo de ação ainda não estar totalmente esclarecido (KENT J, et al., 2019; MC BRAIN RD, et al., 2015). Essa imunoprofilaxia com imunoglobulinas anti-D foi inicialmente usada no pós-natal e tornou-se um protocolo fundamental para prevenção de doenças hemolíticas do feto e do recém-nascido (QURESHI H, et al., 2014).

Desta forma, foi instituído um protocolo de administração pré-natal das preparações anti-Rh(D) do lgG, e que quando combinado com a profilaxia pós-parto padrão erradicou o número de casos. Tal protocolo foi adotado em consenso entre a Faculdade Americana de Obstetras e Ginecologistas (ACOG), a França e a Sociedade Britânica de Hematologia, os quais instituíram em seus respectivos Guidelines (BENNARDELLO F, et al., 2015; AMERICAN COLLEGE OF OBSTETRICIANS AND GYNECOLOGISTS (ACOG), 2017; QURESHI H, et al., 2014).

Com relação à sua distribuição epidemiológica, existe uma escassez de pesquisas acerca deste transtorno e suas respectivas complicações. Sabe-se que as poucas publicações descreveram a prevalência da doença de $R h(D)$ em países de baixa renda, acreditando que nestes países tal agravo não seja considerado um problema de saúde pública devido a uma menor prevalência do tipo sanguíneo $R h(D)$ negativo quando comparados aos países de alta renda (ZIPURSKY A e PAUL VK, 2011; BHUTANI VK, et al., 2013). 
Todavia, estudos epidemiológicos evidenciaram que do total de 134 milhões de nascidos vivos com $\geq$ a 32 semanas de idade gestacional em 184 países (representando 18\%; faixa de incerteza: 23-26 milhões), $480.700(0,36 \%)$ estavam susceptíveis a doença de $\mathrm{Rh}$, e dentre esses $114.100 \mathrm{com}$ risco de morte (24\%; faixa de incerteza: 59.700 172.000), 75.400 com risco de desenvolver kernicterus (16\%) e 52.877 para natimortos (11\%). Três quartos da mortalidade ocorreram na África subsaariana e no sul da Ásia. Além disso, a estimativa de Kernicterus com a doença de Rh variou de 38, 28, 28 e 25/100.000 nascidos vivos para as regiões da Europa Oriental/Ásia Central, África subsaariana, Sul-Asiática e América Latina, respectivamente. E dentre os sobreviventes com kernicterus, mais de $83 \%$ tiveram uma ou mais sequelas (BHUTANI VK, et al., 2013).

Portanto, tornou-se evidente que as mortes neonatais evitáveis ainda ocorrem pela falha em prevenir a sensibilização do Rh, assim como em gerenciar a hiperbilirubemia neonatal. E mesmo havendo comprovação científica da imunoprofilaxia, os protocolos permanecem negligenciados, especialmente em países de baixa renda (BHUTANI VK, et al., 2013).

Quanto à eficácia do protocolo profilático, estudos evidenciaram que após a sua implementação a incidência de sensibilização anti-D reduziu da faixa de $13 \%$ a $19 \%$ para $0,9 \%$ a $1,8 \%$ quando realizado no pós-parto, e para $0,1 \%$ a $0,3 \%$ com a imunoprofilaxia durante o pré-natal, no período compreendido às primeiras 28 semanas (DAJAK S, et al., 2014).

Porém, a profilaxia pré-natal anti-D de rotina não dispõe de um consenso em todo o mundo, sendo recomendada apenas em alguns países, enquanto que em outros a sua utilização ocorre no pós-parto. Além disso, a dose de injeção difere em alguns países devido à falta de imunoglobulina disponível. E por outro lado, quando em doses excessivas podem aumentar o risco de reações alérgicas e doenças infecciosas (XIE X, et al., 2020).

Com isso, a fim de padronizar um protocolo de imunoprofilaxia com IgG anti $R h(D)$ para mulheres $R h D$ negativa, diversas associações de profissionais de saúde envolvidas na prevenção e gestão de Doenças Hemolíticas e Fetos de Recém-nascidos (HDFN), incluindo obstetras e ginecologistas, pediatras e neonatólogos, hematologistas e especialistas em medicina de transfusão, determinaram o uso de imunoglobulinas com 28 semanas de gestação durante cada gravidez, imediatamente após o parto de cada $\mathrm{Rh}(\mathrm{D})$ positivo neonato, e no contexto de qualquer outro evento que possa expô-la ao antígeno $\mathrm{Rh}(\mathrm{D})$, por exemplo, aborto, trauma abdominal (SPERLING JD, et al., 2018). Outra definição determinada no protocolo foi a não administração pré-natal anti-D IgG quando o pai também for $R h(D)$ negativo ou se o feto for digitado com sucesso para o status $\mathrm{Rh}(\mathrm{D})$ por teste de DNA livre de células pré-natal usando plasma materno (DE HAAS M, et al., 2014; DE HAAS M, et al., 2015).

Desta forma, mediante aos resultados de sucesso, à sua eficácia e ao perfil seguro, a imunoglobulina antiD tornou-se um grande avanço preventivo na obstetrícia, todavia seu protocolo imunoprofilático ainda não é utilizado de forma correta em algumas maternidades. Portanto, o objetivo do presente trabalho foi determinar a prevalência de sensibilização ao Rh (D) no período de 2018 a 2020 em uma maternidade pública da cidade de Manaus - Amazonas.

\section{MÉTODOS}

Foi realizado um estudo epidemiológico observacional e descritivo retrospectivo de casos de sensibilização materna a $\operatorname{Rh}(\mathrm{D})$ atendidos em uma maternidade pública no período de 2018 a 2020 na cidade de Manaus, Amazonas. Foram utilizados dados secundários a partir do prontuário das pacientes atendidas na referida maternidade e no tempo predeterminado de 2018 a 2020. As variáveis de estudo para análise foram tipagem sanguínea e fator $\mathrm{RhD}$, idade, raça, idade gestacional, paridade, testes de controle Coombs.

A amostra foi composta pelos prontuários de gestantes catalogados no Serviço de Atendimento Médico e de Emergência (SAME) da maternidade no período de estudo. Foi realizada uma análise aleatória de 39.724 prontuários do total de 95.017 gestantes atendidas na maternidade no período entre 2018 e 2020, para fins de obtenção de um número considerável de casos. 
O projeto foi aprovado pelo Comitê de Ética em Pesquisa da Universidade do Estado do Amazonas (CEP/UEA) sob CAAE: 44083521.8.0000.5016. Foi realizada uma análise estatística descritiva e exploratória dos dados através de frequência relativa.

\section{RESULTADOS}

A fim de obter um número significante de casos de isoimunização foi realizada uma análise aleatória de 39.724 prontuários de gestantes atendidas entre 2018 e 2020, verificando-se uma prevalência de 1,49\% de casos de sensibilização notificados em uma maternidade pública da cidade de Manaus.

A média de idade das 265 gestantes analisadas foi de 25,17 anos e dentre elas $63 \%$ se declararam como parda, 20,4\% como amarela, 9,4\% como branca, $0,8 \%$ como preta e $6,4 \%$ não foram informadas nos prontuários. Quanto à tipagem sanguínea, $61,2 \%$ das mulheres tinham sangue $O$ negativo, 26,3\% com sangue $A$ negativo, 10,2\% com sangue $B$ negativo e apenas 2,3\% com sangue $A B$ negativo. Em contrapartida, os recém-nascidos apresentaram quanto à sua tipagem sanguínea $4,35 \%$ com sangue $A$ negativo, $16,6 \%$ com sangue $A$ positivo, $0,37 \%$ com sangue $A B$ negativo, 2,64\% com sangue $A B$ positivo, $1,13 \%$ com sangue $B$ negativo, 5,7\% com sangue $B$ positivo, $11,7 \%$ com sangue $O$ negativo e $41,5 \%$ com sangue $O$ positivo (Gráfico 1). Porém, um total de $15,1 \%$ de recém-nascidos não teve suas tipagens sanguíneas registradas nos prontuários e os demais $0,73 \%$ foram referentes aos casos de aborto e morte fetal.

Gráfico 1 - Tipagem Sanguínea das Mães e Recém-nascidos atendidos em uma maternidade pública de Manaus entre 2018 e 2020.

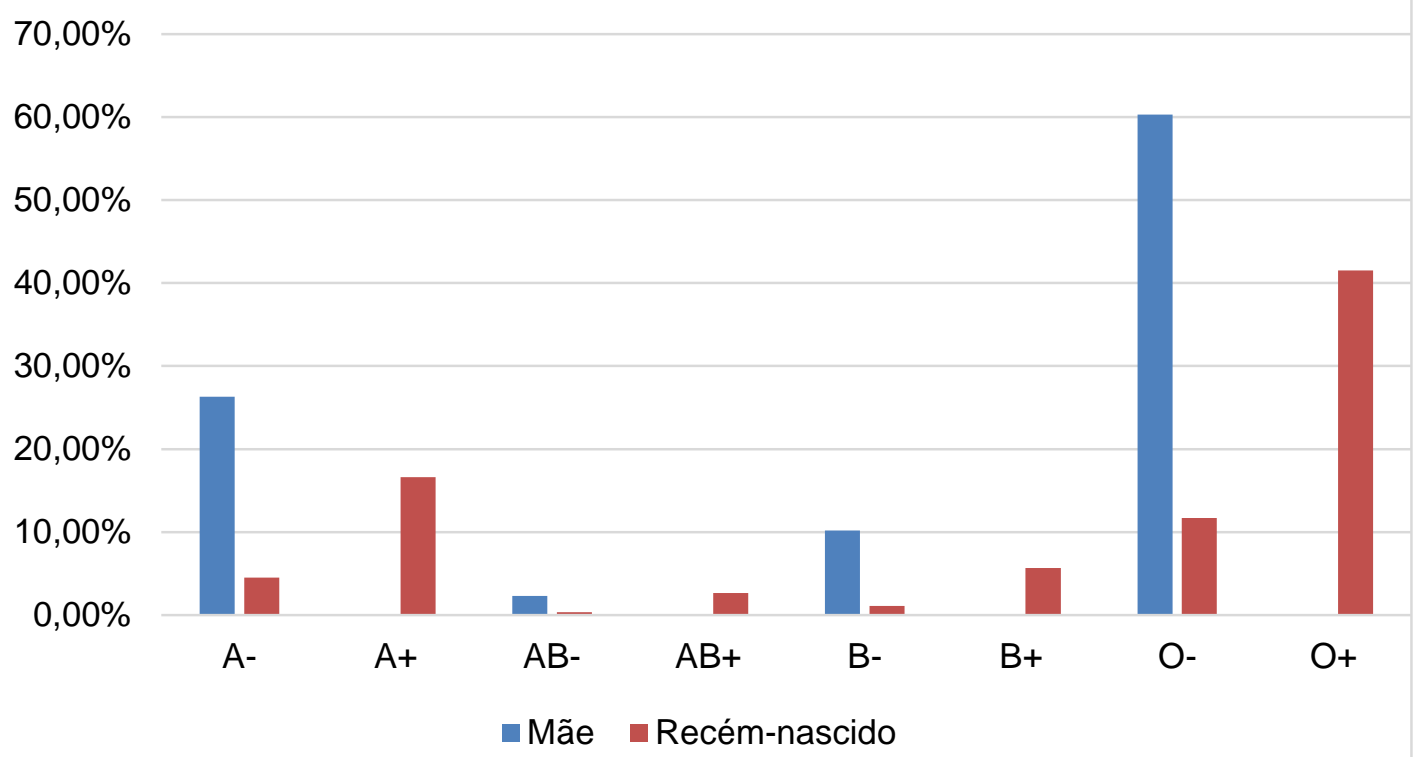

Fonte: Fernandes AP, et al., 2021; dados extraídos de prontuários do Serviço de Atendimento Médico e de Emergência (SAME) da maternidade pesquisada.

A média de consultas de pré-natal realizadas por essas gestantes durante o período gestacional foi de 5,3 consultas e a média de gestações prévia foi equivalente a 2,5 , enquanto o número médio de desfechos convertidos em partos dessas gestações foi de apenas 1,3. A fim de entender melhor a diferença significante no desfecho das gestações, verificou-se que $18,11 \%$ das gestantes relataram um aborto prévio, 3,01\% relataram dois abortos prévios, $0,75 \%$ relataram quatro abortos prévios, e um total de $78,13 \%$ não relatou nenhum caso de aborto prévio.

Com relação às gestações com notificações de isoimunização, verificou-se uma média de 37 semanas gestacionais, sendo $47,5 \%$ dos desfechos com partes cesarianos, sendo dois casos de gemelares notificados, $50,9 \%$ de desfechos com partos normais e $1,6 \%$ de desfechos com abortos. 
Ao analisar acerca da realização do teste de Coombs indireto, um total de $75,5 \%$ das gestantes não realizou este exame, enquanto somente $24,5 \%$ fizeram o teste recomendado. E sobre a administração da imunoglobulina durante o pré-natal $58,8 \%$ das gestantes receberam a profilaxia e em $41,2 \%$ o protocolo profilático não foi realizado. Quando analisada a justificativa das gestantes não terem recebido a imunoprofilaxia, os resultados foram diversos e segue demonstrado abaixo (Gráfico 2).

Gráfico 2 - Justificativas notificadas em prontuário para a ausência de administração de imunoglubulina.

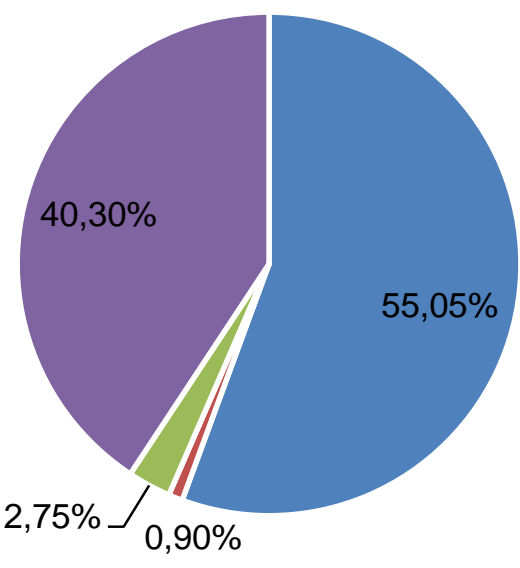

- Nenhum Informação no prontuário

- Farmácia não disponibilizou

- Não havia a Imunoglobulina na Maternidade

- RN com RH negativo

Fonte: Fernandes AP, et al., 2021; dados extraídos de prontuários do Serviço de Atendimento Médico e de Emergência (SAME) da maternidade pesquisada.

\section{DISCUSSÃO}

Apesar da administração profilática de imunoglobulina ter sido descoberta há mais de cinco décadas e desde então utilizada como a terapia padrão na prevenção de sensibilização materna de $R h(D)$ em mulheres $R h(D)$-negativas, os resultados de estudos recentes são decepcionantes (DE HAAS M, et al., 2014). Tais estudos demonstram que os esforços mundiais na prevenção de isoimunização estão muito abaixo do limiar mínimo e distantes do objetivo ideal (DE HAAS M, et al., 2015). Enquanto nos países de baixa renda há uma lacuna entre a disponibilidade e a demanda de Imunoglobulina anti Rh(D), em contrapartida em países de alta renda, apesar da grande oferta, os níveis estão aquém do esperado para fins de prevenção completa (HENDRIGKSON JE e DELANEY M, 2016).

Anualmente, em todo o mundo, estudos constataram que menos de quatro milhões de doses são administradas, índice muito abaixo para prevenção da sensibilização pelo Rh-D, evidenciando que a prevenção completa está distante de ser alcançada, além de desigualdades discrepantes entre países. Sabese que a imunoprofilaxia pré-natal e pós-parto chega a apresentar aproximadamente $99 \%$ de eficácia e que por falta de conhecimento, acesso e mesmo disponibilidade de imunoglobulina mulheres ainda apresentam sensibilização (PEGORARO V, et al., 2020).

Embora não haja um consenso dentre as diversas diretrizes estabelecidas acerca da imunoprofilaxia, recomenda-se que a imunoglobulina anti $R h(D)$ seja administrada em gestante $R h(D)$-negativas durante a $28^{a}$ semana de gestação, imediatamente após o parto de cada recém-nascido $R h(D)$-positivo, bem como em qualquer evento que possa expor essa gestante ao antígeno $\mathrm{Rh}(\mathrm{D})$, seja por algum aborto, traumatismo, abdominal ou acidente (SPERLING JD, et al., 2018; BENNARDELLO F, et al., 2015). 
Porém neste estudo verifica-se que a média de consultas de pré-natal dos casos de isoimunização analisados é de apenas 5,3 consultas, um número muito abaixo do recomendado pelo Ministério da Saúde para que haja um correto planejamento gestacional, bem como prevenção de possíveis anomalias como doenças hemolíticas por incompatibilidade $\mathrm{Rh}$. Este dado pode ser o dado mais relevante no que tange à falta de imunoprofilaxia pré-natal de gestante com possibilidades de sensibilização, uma vez que os testes e acompanhamento gestacional, bem como a administração de imunoglobulina ocorre na $28^{a}$ semana. Logo, a explicação sobre a importância do pré-natal e todas as consequências para o bebê e para a mãe deve ser realizada logo na primeira visita.

A distribuição do complexo Rh é variável entre as diversas populações mundiais, na população brasileira especificamente, a frequência de RhD negativos possui uma estimativa de 10-11\%. Uma vez que a o Brasil possui uma heterogeneidade étnica, poucos indivíduos são verdadeiramente $\mathrm{RhD}$ negativo, dos quais os eritrócitos realmente não transportam nenhum antígeno $D$. Sendo assim, nenhuma outra ação é justificada em termos de cuidados clínicos pré-natais (CRUZ BR, et al., 2012; ZIZA KC, et al., 2017).

No que tange a população afrodescendente, a frequência corresponde a $92 \%$ e em algumas populações do extremo oriente chega a $99 \%$. Consequentemente os indivíduos RhD negativo compreendem um percentual muito inferior a este número e entre os brasileiros a estimativa é menor que $15 \%$. Entre casais brancos estima-se uma frequência de $10 \%$ da presença de incompatibilidade, enquanto entre casais negros esse valor corresponde a $7,8 \%$, valores já corrigidos quanto à heterogeneidade do genoma. Por outro lado, a porcentagem de pessoas com sangue Rh negativo na América do Norte e na Europa é de aproximadamente 15\%, afro-americanos de $8 \%$, ascendentes chineses $0,3 \%$ e indígenas 5\% (IZETBEGOVIC S, 2013).

Sendo assim, o baixo número de isoimunização por incompatibilidade encontrado no estudo de 1,6\% pode ser justificado pela predominância de mulheres que se declaram pardas no Amazonas, ou seja, uma população que possui características predominantes de indígenas e negros. Portanto, a predominância de Rh positivo na população amazonense corrobora com uma prevalência relativamente baixa, porém vai de encontro ao número de casos ainda significantes em uma região na qual a isoimunização poderia estar devidamente controlada.

Apesar das taxas mais baixas encontradas nas regiões Norte e Nordeste, principalmente associado a menor proporção de mulheres brancas e por conseguinte de Rh negativos, elas ainda estão acima daquela esperada se a profilaxia estivesse sendo feita sem falhas. Além disso, existe a grande possibilidade de subnotificações que dificulta ainda mais o controle de possíveis falhas na implementação da imunoprofilaxia.

Portanto, torna-se imprescindível a realização dos testes de coombs, que permite reconhecer a fração Fc do anticorpo fixado na membrana das hemácias sensibilizadas. Portanto, uma ligação entre os anticorpos humanos, resultando no fenômeno da aglutinação (VIZZON AG e SILVA, FRM, 2015). Porém, neste estudo verificou-se que um total de $75,5 \%$ das gestantes não realizou este exame, enquanto somente $24,5 \%$ fizeram o teste recomendado. Sem que houvesse registro em prontuários dos motivos pelos quais uma grande quantidade de mulheres não realizara o exame.

Acerca do protocolo fundamentado de profilaxia com imunoglobulina anti-D, o presente estudo apresentou um total de 58,8\% de casos que não receberam a imunoprofilaxia conforme a indicação do protocolo. Quando analisados os motivos, observam-se notificações em prontuários médicos como: falta de imunoglobulina na maternidade; não autorização do setor de farmácia; ou mesmo a maioria que não tinha nenhuma informação. Seja qual for o motivo, nenhum justifica a negligência de um protocolo que já está consolidado em todo o mundo. Portanto, tornou-se evidente que as mortes neonatais evitáveis ainda ocorrem pela falha em prevenir a sensibilização do Rh, assim como em gerenciar a hiperbilirrubemia neonatal. $E$ mesmo havendo comprovação científica da imunoprofilaxia, os protocolos permanecem negligenciados, especialmente em países de baixa renda (BHUTANI VK, et al., 2013).

Acerca das doenças hemolíticas perinatais, um estudo prospectivo verificou que em 4.321 gestações saudáveis houve uma taxa de prematuridade de $5 \%$, sendo que esses recém-nascidos prematuros apresentaram seus níveis de bilirrubina alterados, elevando o risco de mortalidade bem como de comprometimento neurológico durante seu desenvolvimento (VOGEL JP, et al., 2018). E dentre os 
tratamentos indicados para os casos de eritroblastose fetal associada à incompatibilidade de Rh, o mais comumente utilizado é a fototerapia intensiva. Nesses casos, deve haver um monitoramento contínuo e seriado para avaliar a concentração de bilirrubina sérica a fim de obter os valores aceitáveis (UWINGABIYE J, et al., 2016).

Corroborando com os resultados deste estudo, verificou-se que ao avaliar a incompatibilidade ABO entre prematuros e a causa de maiores incidências de reação hemolítica, incluindo anemia e hiperbilirrubinemia, ainda não é possível estabelecer uma relação direta entre esses desfechos gestacionais de prematuridade e possíveis complicações de doenças hemolíticas em recém-nascidos (YOGEV-LIFSHITZ M, et al., 2016). Porém, por consensos e opiniões de especialistas do Brasil, recomenda-se administrar imunoglobulina anti$\mathrm{D}$ em todos os casos de abortamentos com intervenção cirúrgica, independentemente da idade gestacional. E apesar de não haver nenhum estudo que comprove a relação direta da sensibilização materna com a prematuridade, verificou-se que um total de $1,6 \%$ casos dentre as gestações avaliadas apresentaram casos de abortos, ressaltando a necessidade de estudo mais consistente para possível correlação.

Levando em consideração que a incompatibilidade $\mathrm{Rh}(\mathrm{D})$ entre mão e feto seja a principal causa de doenças hemolíticas em recém-nascidos, como a eritroblastose fetal, podendo comprometer o desfecho da gravidez, ou mesmo possuir sequelas inerentes à isoimunização materna, tais como graves lesões no sistema nervoso. Os fatores que culminam nesse evento durante a gestação estão relacionados diretamente com falhas na imunoprofilaxia durante a gravidez, ou ainda falha na administração da imunoglobulina após o parto de uma criança Rh positiva. (SIMÃO MCSA, et al., 2021). Este estudo corrobora com os dados encontrados no presente estudo, principalmente, acerca da falha na administração de imunoglobulina anti-D, que foi evidenciado nas notificações de prontuários.

Dado o exposto, o presente estudo recomenda a elaboração de um fluxo de atendimentos e de condutas para gestantes com riscos de isoimunização, para que desta forma haja a erradicação das doenças hemolíticas neonatais, uma vez que existe um protocolo eficaz e comprovado em todo mundo. Além disso é importante realizar palestras a fim de mostrar a importância do pré-natal, especialmente para as gestantes com isoimunização, e desta forma supervisionar e controlar o período gestacional e perinatal.

\section{CONCLUSÃO}

O presente estudo concluiu que a taxa de prevalência de isoimunização na maternidade pública de Manaus, apesar de baixa poderia irrelevante, uma vez que a maioria das mulheres na Região Norte não apresenta tipo sanguíneo Rh negativo. A subnotificação e falta de preenchimento correto dos prontuários podem ser as principais causas na falha na implementação universal do protocolo de imunoprofilaxia anti-D, bem como a defasagem das consultas de pré-natal, comprometendo diretamente na prevenção de doenças perinatais. E após o desfecho de gestação não há um campo em prontuários para registro de condutas pósnatais, seja em casos de abortamentos, cesarianos e vaginais. Sendo assim, visando melhorar a eficácia da prevenção, sugerimos a implementação de um fluxo de atendimentos e de registros na maternidade para que os casos sejam devidamente notificados, assim como a conduta realizada em cada paciente para fins estatísticos e de controle das doenças hemolíticas perinatais.

\section{REFERÊNCIAS}

1. AMERICAN COLLEGE OF OBSTETRICIANS AND GYNECOLOGISTS (ACOG) practice bulletin No. 181: Prevention of Rh $\mathrm{D}$ alloimmunization. Clinical management guidelines for obstetrician-gynecologists. Obstetrics \& Gynecology, 2017; 130(2): 57-70.

2. BENNARDELLO F, et al. Recommendations for the prevention and treatment of haemolytic disease of the foetus and newborn. Blood Transfusion, 2015; 13(1): 109- 34.

3. BHUTANI VK, et al. Neonatal hyperbilirubinemia and Rhesus disease of the newborn: incidence and impairment estimates for 2010 at regional and global levels. Pediatric Research. 2013; 74(S1): 86-100.

4. CRUZ BR, et al. RHD alleles in Brazilian blood donors with weak D or D-negative phenotypes. Transfus Med, 2012; 22: 84-89. 
5. DAJAK S, et al. The importance of antenatal prevention of RhD immunisation in the frst pregnancy. Blood Transfus, $2014 ; 12(3): 410$.

6. DE HAAS M, et al. Anti-D prophylaxis: past, present and future. Transfusion Medicine, 2014; 24(1): 1-7.

7. DE HAAS M, et al. Haemolytic disease of the fetus and newborn. Vox Sanguinis, 2015; 109(2): 99-113.

8. HENDRIGKSON JE, DELANEY M. Hemolytic disease of the fetus and newborn: modern practice and future investigations. Transfusion Medicine Reviews. 2016; 30(4):159-64.

9. IZETBEGOVIC S. Occurrence of $\mathrm{ABO}$ and RhD incompatibility with Rh negative mothers [Internet]. Vol. 25, Mater Sociomed. 2013. p. 255-8.

10. QURESHI E, et al. BCSH guideline for the use of anti-D immunoglobulin for the prevention of haemolytic disease of the fetus and newborn. Transfusion Medicine, 2014; 24: 8-20.

11. KENT J, et al. Routine administration of AntiD: the ethical case for offering pregnant women fetal RHD. The Journal of Obstetrics and Gynecology of India, 2019; 69(5):420-425.

12. MC BRAIN RD, et al. Anti-D administration in pregnancy for preventing Rhesus alloimmunisation. Cochrane Database Syst Rev., 2015; 9(2).

13. NASSAR GN, WEHBE C. Erythroblastosis Fetalis. In: STATPEARLS: Content is King [Internet]. Treasure Island: StatPearls Publishing, 2020.

14. PEGORARO V, et al. Hemolytic disease of the fetus and newborn due to $R h(D)$ incompatibility: A preventable disease that still produces significant morbidity and mortality in children. PLoS One, San Francisco, 2020; 15(7).

15. QURESHI H, et al. BCSH guideline for the use of antiD immunoglobulin for the prevention of haemolytic disease of the fetus and newborn. Transfusion Medicine, 2014; 24(1): 8-20.

16. SARWAR A, SRIDHAR DC. Rh-Hemolytic Disease. In: STATPEARLS: Content is King [Internet]. Treasure Island: StatPearls Publishing, 2020.

17. SIMÃO MCSA, et al. Prognóstico de Eritroblastose Fetal em Crianças Prematuras. Brazilian Journal of Health Review, 2021; 4(2): 4602-4618.

18. SPERLING JD, et al. Prevention of RhD alloimmunization: a comparison of four national guidelines. American Journal of Perinatology, 2018; 35(02): 110.

19. UWINGABIYE J, et al. Severe hemolytic disease of the premature newborn due to $\mathrm{RH} 1$ incompatibility: a case report. Clujul Med., Cluj-Napoca, 2016; 89 (4): 565-568.

20. VIZZON AG, SILVA FRM. Teste da antiglobulina humana: uma revisão de literatura. Revista Eletrônica de Farmácia / Eletronic Journal of Pharmacy, 2015; 12(3): 5-14.

21. VOGEL JP, et al. The global epidemiology of preterm birth. Best Pract Res Clin Obstet Gynaecol., 2018; 52: 3-12.

22. XIE X, et al. Clinical value of different anti-D immunoglobulin strategies for preventing Rh hemolytic disease of the fetus and newborn: A network meta-analysis. PLoS ONE, 2020; 15(3).

23. YOGEV-LIFSHITZ M, et al. Indication of Mild Hemolytic Reaction Among Preterm Infants With ABO Incompatibility. Pediatr Blood Cancer, 2016; 63(6): 1050- 1053.

24. ZIPURSKY A, PAUL VK. The global burden of Rh disease. Archives of Disease in Childhood-Fetal and Neonatal Edition, 2011; 96(2): F84-5.

25. ZIZA KC, et al. Determination of Fetal RHD Genotype Including the RHD Pseudogene in Maternal Plasma. J Clin Lab Anal, 2017; 31 (3): e22052. 